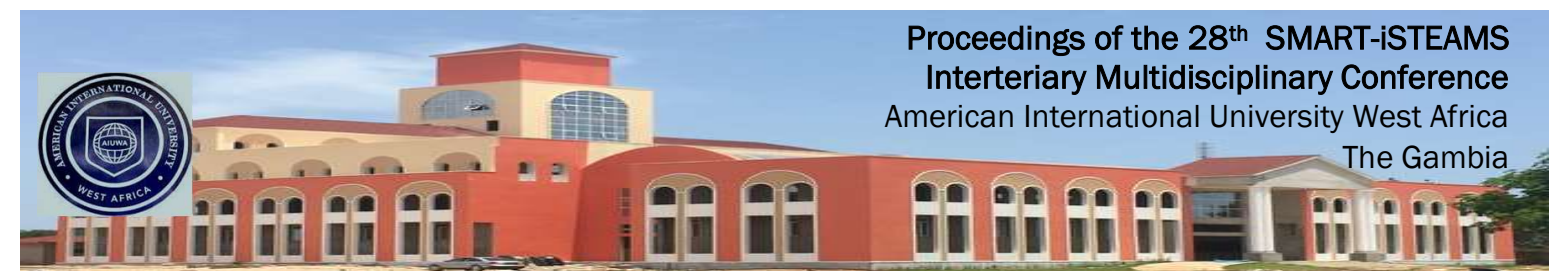

\author{
Full Research Paper
}

\title{
Magnetohydrodynamic Flow and Heat Transfer Characteristics in Micropolar-Casson Fluid over a Stretching Surface with Temperature-dependent Material Properties.
}

Fatunmbi, E.O.

Akanbi, 0.0.

Dept. of Maths \& Statistics

Federal Polytechnic

Ilaro, Nigeria.

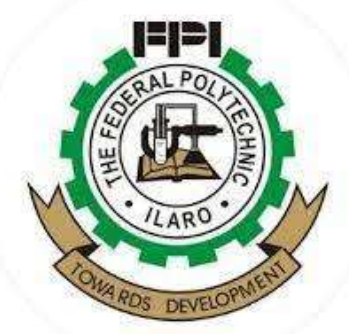

E-mails

ephesus.fatunmbi@federalpoly ilaro.edu.ng,

olumuyiwa.akanbi@federalpoly

ilaro.edu.ng

Phone

+2348034013598 ;

$+2347033549098$

\begin{abstract}
The current investigation communicates the flow and heat transfer characteristics of an electrically conducting micropolar-Casson fluid over a two-dimensional stretching surface with variable thermal conductivity and viscosity. Thermal radiation, viscous dissipation and heat source effects are also accounted for in the energy equation. The formulated equations of flow and heat transfer are converted from partial to ordinary differential equations using suitable similarity transformations while the dimensionless equations are solved by Runge-Kutta Fehlberg integration scheme. The effects of the physical parameters are publicized through graphs and validated by related published studies in the limiting situations. It is found from the investigation that there is accelerated flow due to the material micropolar term whereas the presence of Casson fluid and magnetic field terms decelerate the velocity. Besides, the surface temperature improves with a rise in the Casson fluid term, Eckert number and thermal conductivity parameter whereas the trend is reversed for micropolarity influence.
\end{abstract}

Keywords: Micropolar-Casson fluid; Magnetohydrodynamic, Variable viscosity; Variable thermal conductivity; Viscous dissipation

Proceedings Reference Format

Fatunmbi, E.O. \& Akanbi, O.O. (2021): Magnetohydrodynamic Flow and Heat Transfer Characteristics in Micropolar-Casson Fluid over a Stretching Surface with Temperature-dependent Material Properties. Proceedings of the 28th iSTEAMS Intertertiary Multidisciplinary Conference. American Int University West Africa, The Gambia. October, 2021. Pp 97-112. www.isteams.net/gambia2021. DOI - https://doi.org/ 10.22624/AIMS/iSTEAMS-2021/V28N2P7 


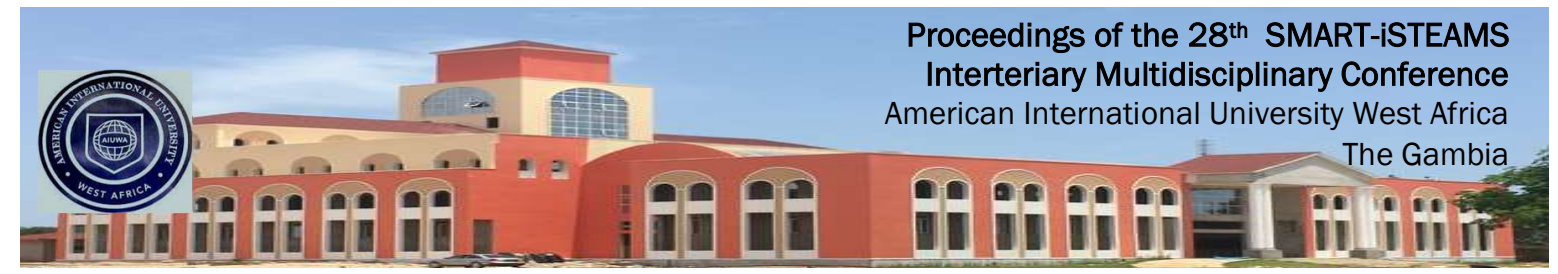

\section{INTRODUCTION}

In recent times technology has permeated every facet of life even the classroom is not left out. The use of whatsapp, facebook, Twitter Telegram, Instagram is on the increase. This uction Fluid flow and heat transfer processes can be altered by the imposition of magnetic field in the flow regime. Magnetohydrodynamics (MHD) deals with the interaction of magnetic field and electrically conducting fluids. The application of such flow in diverse fields of applied sciences and engineering are huge. For instance, in metallurgical industries, magnetic field can be applied to heat up, pump, stir, levitate liquid metals and for the purification of molten metals from non-metallic inclusions, It is also useful in plasma, nuclear reactors, MHD generators and accelerators, boundary layer control in aerodynamics (Ibrahim and Makinde, 2015).

The boundary layer flow of MHD over a stretching sheet has been reported by many researchers under different assumptions, configurations and category of fluids (see Qasim, 2013; Ahmad, et al., 2016; Mahanthesh et al., 2018; Ullah et al. 2020; Fatunmbi and Adeniyan, 2020). The simple micro-fluids theory formulated by Eringen (1964) characterizes fluids with microconstituents. The theory deals with isotropic viscous fluids with micro-elements and micromotion which manifest certain microscopic influences arising from the local structure and micromotion of the fluid parcels. Besides, these fluids have the ability to support stress and body moments with the effects of rotation inertia. For the application of simple micro-fluid concept to the cases of real flow situations, Eringen (1966) simplified further the concept of simple micro-fluids to formulate a subclass of microfluids known as micropolar fluid. Micropolar fluid characterizes fluids with rigid, randomly oriented particles suspended in a viscous medium such as polymeric fluids, liquid crystals, where particles deformation is neglected (Lukaszewicz, 1999).

These rigid particles contained in a small volume can spin about the centroid of the volume element. It also defines a substantial generalization of the Navier-Stokes model and open up a new field of potential applications in extrusion of polymer fluids, the cooling of metallic plate in water bath, synovial lubrication, arterial blood flows, sediment transport in rivers, etc. (Rahman, 2009; Reena and Rana, 2009). Fluids that can be categorized as micropolar fluids are polymeric fluids, fluid suspensions, animal blood, liquid crystals, colloidal fluids, etc (Ahmadi, 1976; Hayat, Mustafa and Obaidat, 2011). The flow and heat transfer of micropolar fluid have been studied by many researchers on different geometries, assumptions and conditions (see Mahmoud, 2007; Salawu and Fatunmbi, 2017; Keimanesh and Aghanajafi, 2017; Fatunmbi and Adeniyan, 2018; Fatunmbi and Okoya, 2020).

The Casson fluid model describes a shear thinning fluid which exhibits yield stress attribute. It posses a property of infinite viscosity at zero rate of shear stress and zero viscosity at infinite rate of shear stress, Das et al. (2018). Whenever the yield stress is greater than the shear stress the fluid characterizes solid nature but when the yield stress is lower than the applied shear stress the fluid begins to flow. This model was invented by Casson (1959) while investigating a flow equation for pigment oil-suspensions of printing ink. Bird et al. (1983) studied the rheology and transport of visco-plastic materials and communicated the fact that the concept of Casson fluid fits a plastic fluid model with shear thinning attributes. 


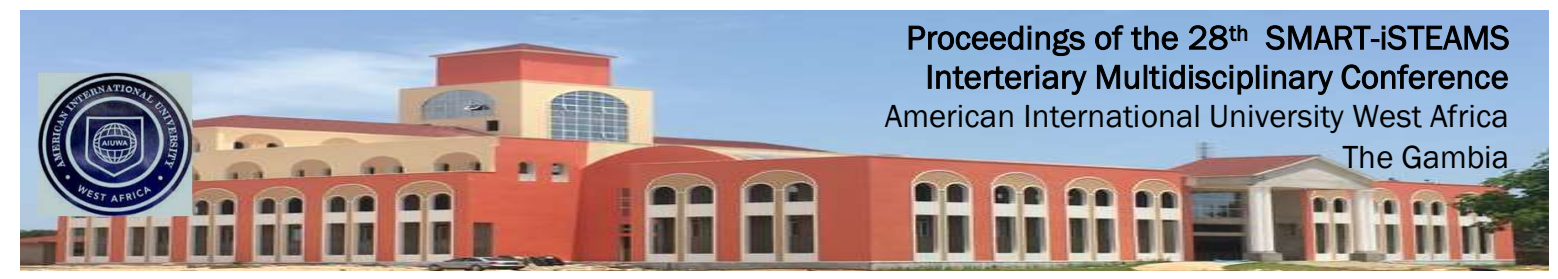

This model has become prominent among other non-Newtonian fluids due to its consequential applications. The suitability of this model to adequately describe the rheological behaviour of various ingredients as paints, lubricants, jelly,tomato sauce, blood, honey, etc has been investigated researchers Casson (1959). For low shear rates, the Casson fluid simulates accurately the flow attributes of blood. Also, the manifestation of protein, fibrinogen as well as globulin in aqueous base plasma, red blood cells portrays human blood as a good example of Casson fluid. These applications have aroused the interest of researchers and scientists to study such a fluid on various configurations and conditions.

Vajravelu et al. (2016) analytically reported a mixed convective motion of Casson fluid configured in a vertically stretched sheet with non-uniform thermal conductivity and prescribed surface temperature condition. Das et al (2018) examined the transport of Casson fluid over an exponentially stretching sheet with the impact of thermal radiation and entropy generation while Krishna et al (2018) analyzed the motion of a reactive Casson fluid in a porous stretching surface. The authors reported that the reactions of Casson parameter and magnetic field term are similar in respect to the velocity profile. Recently, An investigation of hydromagnetic Casson nanofluid in a porous medium was conducted by Fatunmbi and Okoya (2021) with the impact of nonlinear Boussinesq approximation and variable thermal conductivity near a stagnation point. It was pointed out that the Casson fluid material term enhances the thermal field and improved the viscous drag. Likewise, the motion of a radiative Casson fluid over a convectively heated permeable sheet with the impact of Joule heating and wall slip was recently examined by Omotola and Fatunmbi (2021).

The blend of micropolar and Casson fluid properties can be referred to as the micropolar-Casson fluid. Such a composition becomes so crucial in applications particularly in bio-engineering processes, metallurgy, food production, production of pharmaceutical products, paints, synthetic lubricants, biological fluids, e.g. blood flow in human body and drilling operations. Mehmood et al. (2017) numerically investigated such a blend over a convectively heated stretching material with internal heat source whereas the examination of such mixture over a stretching surface characterized by inclined magnetic field and viscous dissipation was done by lqbal et al. (2017). It was stated by the authors that micropolar material parameter raises the heat transfer and the viscous drag. The aforementioned authors however studied the case of uniform viscosity and thermal conductivity. For better and accurate prediction of the flow behaviour, it is imperative to incorporate temperature-dependent flow properties.

The aim of this study therefore is to investigate the flow of an electrically conducting micropolarCasson fluid over a stretchable material with the effects of temperature-dependent viscosity and thermal conductivity, viscous dissipation and internal heat generation in the presence of isothermal wall condition. The applications of this study to various areas in industries and engineering as mentioned above have induced its investigation. The main equations are solved numerically via Runge-Kutta Fehlberg scheme while the results are presented in tables and graphs with appriopriate discussion.. 


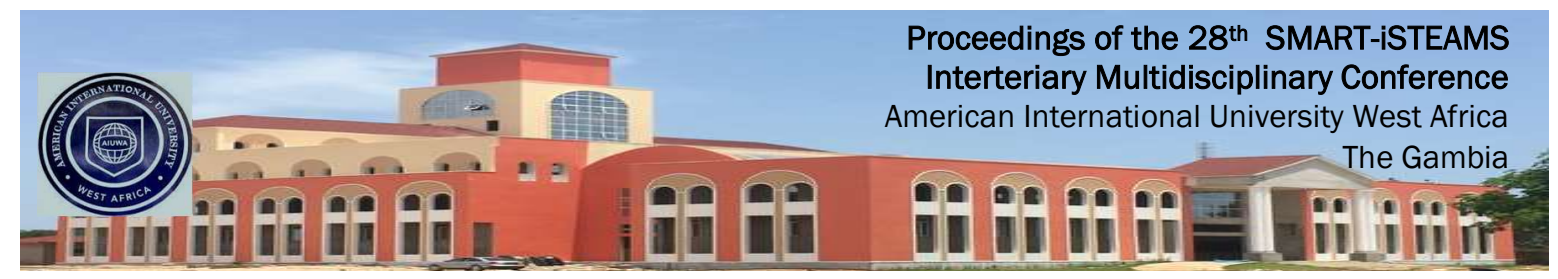

\section{PROBLEM FORMULATION}

Consider, an incompressible and steady flow and heat transfer characteristics of an electrically conducting micropolar-Casson nanofluid flow configured in a two-dimensional stretching material in a porous medium. Taking the coordinate as $(x, y)$ having a corresponding velocity components $u$ and $v$ where the flow is directed towards the $x$ axis with $y$ axis being normal to it as shown in Figure. 1. An external magnetic field of uniform strength is applied normal to the flow direction while the impact of the induced magnetic field and electric field is assumed to be negligible on the account of significantly low Reynolds number. It is assumed that the viscosity and thermal conductivity vary linearly with temperature whereas other fluid properties are assumed to be isotropic and uniform. The impact of thermal radiation, viscous dissipation and internal heat generation are incorporated in the heat equation.

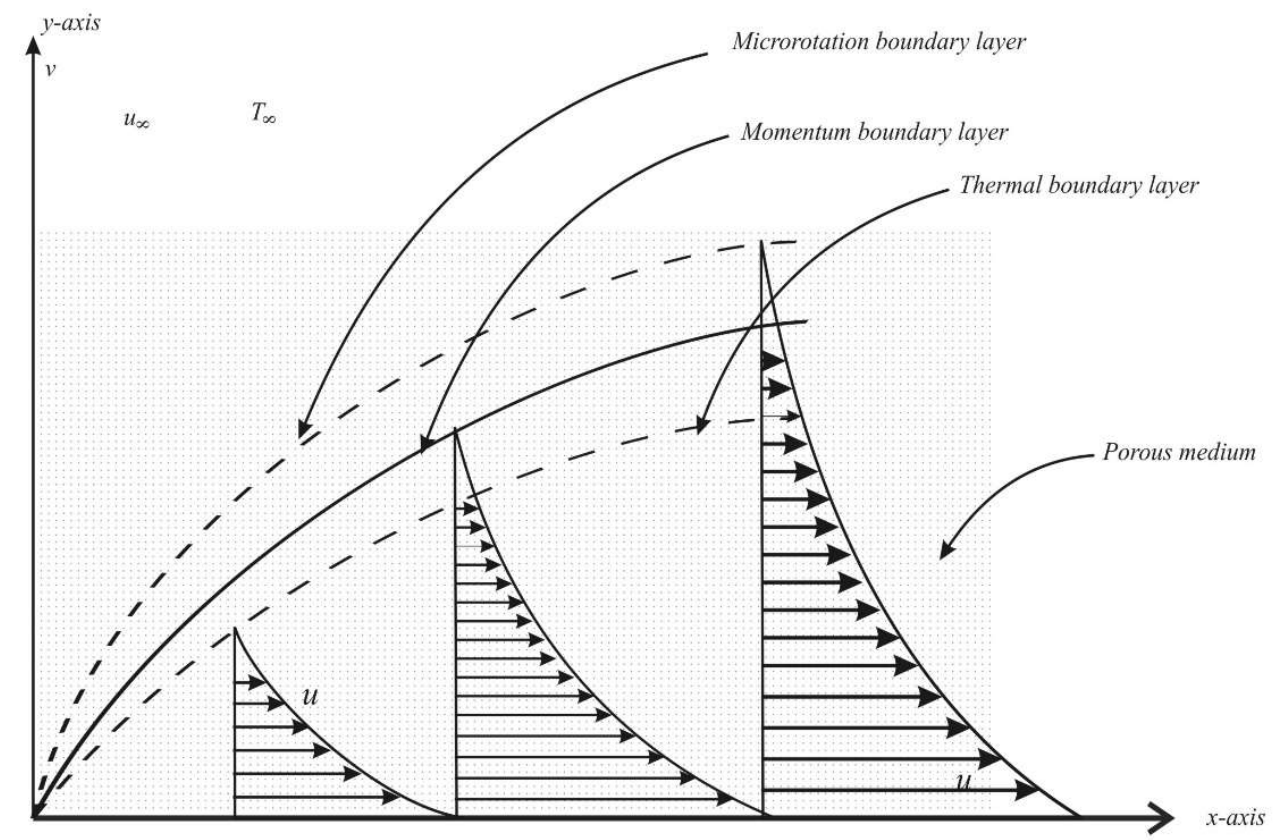

Figure 1. Flow Configuration

The stress tensor and couple stress tensor relations for isotropic micropolar fluid are expressed as (see Eringen, 1966; Lukaszewicz, 1999; Chen et al., 2011):

$$
\begin{gathered}
\tau_{i j}=\left(-P+\lambda_{r} v_{k, k}\right) \delta_{i j}+\mu\left(v_{i, j}+v_{j, i}\right)+\kappa\left(v_{j, i}-v_{i, j}\right)-\kappa \epsilon_{k i j} N_{k}, \\
C_{i j}=c_{o} N_{k, k} \delta_{i j}+c_{d}\left(N_{i, j}+N_{j, i}\right)+c_{a}\left(N_{i, j}-N_{j, i}\right) .
\end{gathered}
$$




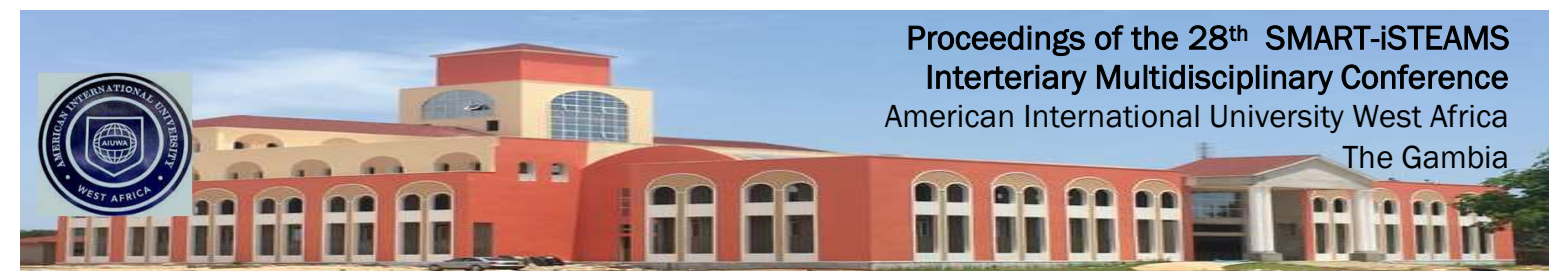

Where $\tau_{i j}^{S}=\left(-P+\lambda_{r} v_{k, k}\right) \delta_{i j}+\mu\left(v_{i, j}+v_{j, i}\right)$ is the symmetric part of the stress tensor $\tau_{i j}$ which denotes the tress tensor for the classical hydrodynamics. Also, $\tau_{i j}$ is the Cauchy stress tensor, $P$ is the pressure, $\lambda_{r}$ and $\mu$ are second viscosity coefficient and dynamic viscosity respectively. $\kappa$ is the dynamic microrotation viscosity, $c_{o}, c_{a}$ and $c_{d}$ are the coefficients of angular viscosity, $v_{i}, N_{k}$ and $\epsilon_{i j k}$ are the velocity component, angular velocity component and the alternating/permutation stress tensor, $C_{i j}$ is the couple stress tensor, $\delta_{i j}$ is the usual Kronecker delta, $v_{i, j}=\frac{\partial v_{i}}{\partial x_{j}}, N_{i, j}=\frac{\partial N_{i}}{\partial x_{j}}$ are the partial derivatives with respect to coordinate $\left(x_{1}, x_{2}, x_{3}\right)$. The following inequalities must hold for Eq. (1) to remain valid: $\mu \geq 0,3 \lambda+2 \mu \geq$ $0, \kappa \geq 0$.

Likewise, the rheological equation of an isotropic, incompressible flow of Casson fluid is specified as

$$
S_{i j}=\left(\mu_{B}+\frac{P_{y}}{\sqrt{2 \pi}}\right) 2 e_{i j} ; \pi>\pi_{c}, \quad S_{i j}=\left(\mu_{B}+\frac{P_{y}}{\sqrt{2 \pi_{c}}}\right) 2 e_{i j} ; \pi<\pi_{c},
$$

where $S_{i j}$ denotes the Cauchy stress tensor, $P_{y}$ stands for the yield stress of the fluid described as

$$
P_{y}=\frac{\mu_{B} \sqrt{2 \pi}}{\beta}
$$

Also, $\mu_{B}$ defines the plastic dynamic viscosity of the non-Newtonian fluid while $\pi$ depicts the product of deformation rate with itself $\left(\pi=e_{i j} e_{i j}\right), \pi_{c}$ denotes the critical value of the product of the component of the deformation rate with itself which is based on the non-Newtonian model. Similarly, one can write

$$
\mu=\mu_{B}+\frac{P_{y}}{\sqrt{2 \pi}}
$$

and on substituting Eq.(4) into (5) results to

$$
\mu=\mu_{B}\left(1+\frac{1}{\beta}\right) \Rightarrow \vartheta=\frac{\mu_{B}}{\rho}\left(1+\frac{1}{\beta}\right)
$$

where $\vartheta$ is the kinematic viscosity and $\beta=\mu_{B} \frac{\sqrt{2 \pi}}{P_{y}}$ describes the Casson fluid parameter. Now, on the assumption that the viscosity and thermal conductivity are temperature-dependent and that the medium is porous, the partial differential equations governing the flow and heat transfer of Micropolar-Casson fluid model can be written as

$$
\begin{gathered}
\frac{\partial u}{\partial x}+\frac{\partial v}{\partial y}=0 \\
\bar{u} \frac{\partial u}{\partial x}+v \frac{\partial u}{\partial y}=\frac{1}{\rho_{\infty}}\left[\left(1+\frac{1}{\beta}\right) \frac{\partial}{\partial y}\left(\mu_{B_{\infty}} \frac{\partial u}{\partial y}\right)\right]+\frac{\kappa}{\rho_{\infty}} \frac{\partial^{2} u}{\partial y^{2}}+\frac{\kappa}{\rho} \frac{\partial N}{\partial y}-\frac{1}{\rho_{\infty}}\left[\frac{\mu_{B_{\infty}}}{K_{p}}\left(1+\frac{1}{\beta}\right)+\kappa\right] u-\frac{\sigma B_{o}^{2}}{\rho_{\infty}} u,
\end{gathered}
$$




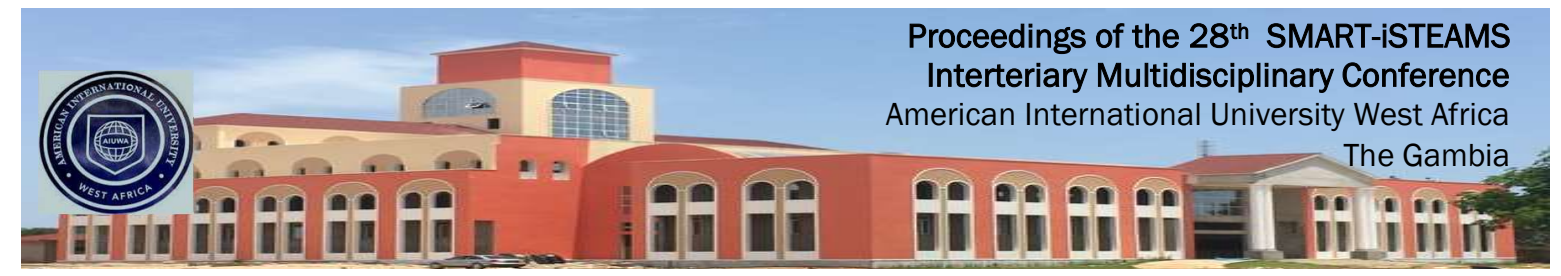

$$
\begin{gathered}
u \frac{\partial N}{\partial x}+v \frac{\partial N}{\partial y}=\frac{\gamma}{\rho_{\infty} j} \frac{\partial^{2} N}{\partial y^{2}}-\frac{\kappa}{\rho_{\infty} j}\left(2 N+\frac{\partial u}{\partial y}\right) \\
u \frac{\partial T}{\partial x}+v \frac{\partial T}{\partial \bar{y}}=\frac{1}{\rho_{\infty} C_{p}} \frac{\partial}{\partial y}\left[\left(k(T)+\frac{16 T_{\infty}^{3} \sigma^{\star}}{3 k^{\star}}\right) \frac{\partial T}{\partial y}\right]+\frac{1}{\rho_{\infty} C_{p}}\left[\mu_{B_{\infty}}(T)\left(1+\frac{1}{\beta}\right)+\kappa\right]\left(\frac{\partial u}{\partial y}\right)^{2}+\frac{Q^{\star}\left(T-T_{\infty}\right)}{\rho_{\infty} C_{p}} .
\end{gathered}
$$

The associated boundary conditions are:

$$
\begin{aligned}
& y=0: u=u_{w}=a x, v=0, N=-n \frac{\partial u}{\partial y}, T=T_{w} \\
& y \rightarrow \infty: u \rightarrow 0, N \rightarrow 0, T \rightarrow T_{\infty}
\end{aligned}
$$

where $u$ and $v$ are the velocity components in $x$ and $y$ directions respectively, $a$ is constant $>$ 0 . Also, $\rho$ is the fluid density, $\kappa$ is the vortex or microrotation viscosity, $T$ is the fluid temperature, $N$ is the component of microrotation whose direction of rotation is in $x y$ plane, $B_{o}$ is the magnetic field intensity, $j$ indicates the microinertia density, $C_{p}$ is the specific heat at constant pressure, $T_{w}$ is the temperature of the stretching sheet, $T_{\infty}$ is the free stream temperature, $q_{r}$ radiative heat flux, $Q^{\star}$ is the volumetric rate of heat generation/absorption and $K_{p}$ is the permeability of the porous medium. Similarly, $n$ is a surface boundary parameter with $0 \leq n \leq$ 1. The case when $n=0$ corresponds to $N=0$, this represents no-spin condition i.e. strong concentration such that the micro-particles close to the wall are unable to rotate.

The case $n=\frac{1}{2}$, indicates weak concentration of micro-particles and the vanishing of antisymmetric part of the stress tensor and the case $n=1$ represents turbulent boundary layer flows. Following previous authors, it is reasonable to consider the temperature-dependent viscosity and thermal conductivity model as considered by Layek et al. (2005); Salem and Fathy (2012); Fatunmbi et al (2020). Hence, the plastic dynamic viscosity and thermal conductivity variation with temperature are expressed respectively given in Eq. (12) as

$$
\mu_{B}(T)=\mu_{B_{\infty}}\left[1+\zeta\left(T_{w}-T\right)\right], k(T)=k_{\infty}\left[1+\epsilon\left(T-T_{\infty}\right)\right]
$$

Here $\mu_{B_{\infty}}$ is the fluid viscosity at reference temperature, $k_{\infty}$ is the thermal conductivity of the fluid far away from the surface sheet, $\zeta$ and $\epsilon$ are constants. Introducing the similarity transformations variables and stream functions (11) into the governing equations.

$$
\eta=y\left(\frac{a}{v_{\infty}}\right)^{1 / 2}, \psi=f(\eta) x(a v)^{1 / 2}, N=\operatorname{axg}(\eta)\left(\frac{a}{v}\right)^{\frac{1}{2}}, \theta=\frac{T-T_{\infty}}{T_{w}-T_{\infty}}, u=\frac{\partial \psi}{\partial y}, v=-\frac{\partial \psi}{\partial x}
$$

Then, the continuity equation (7) is satisfied in view of Eq. (13) while Eqs. (8-10) taking cognizance of Eq. (10) transform to ordinary differential equations as listed below. 


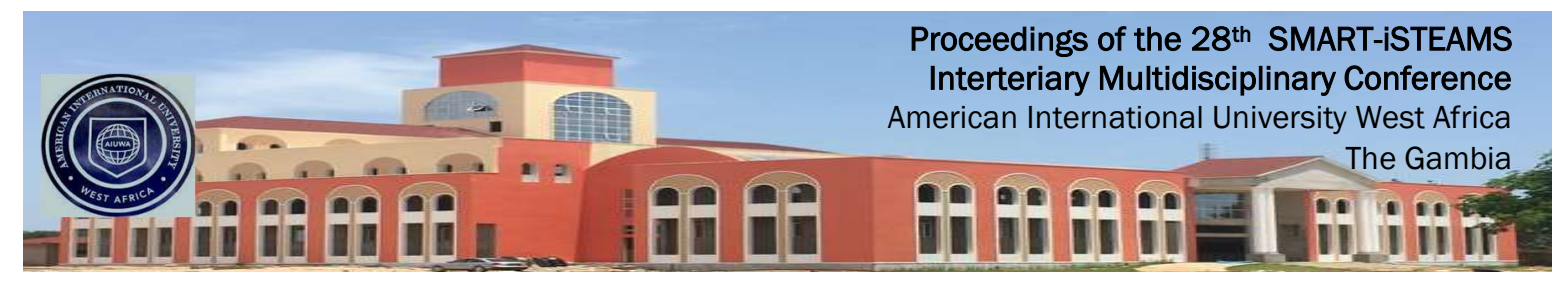

\subsection{The Transformed Equations}

$$
\begin{gathered}
\left(1+\frac{1}{\beta}\right)\left[(1+\xi-\xi \theta+K) f^{\prime \prime \prime}-\xi \theta^{\prime} f^{\prime \prime}-D a(1+\xi-\xi \theta) f^{\prime}-M f^{\prime}\right]-f^{2}+f f^{\prime \prime}+K g^{\prime}=0 \\
(1+K / 2) g^{\prime \prime}+f g^{\prime}-f^{\prime} g-K\left(2 g+f^{\prime \prime}\right)=0 \\
\frac{1}{P r}(1+h \theta+R) \theta^{\prime \prime}+h \theta^{\prime 2}+f \theta^{\prime}+\left(1+\frac{1}{\beta}\right)(1+\xi-\xi \theta+K) f^{\prime \prime 2} E c+B \theta
\end{gathered}
$$

The conditions at the boundary transmute to

$$
\begin{aligned}
& f^{\prime}(0)=1, f(0)=0, g=n f^{\prime \prime}(0), \theta(0)=1 \\
& f^{\prime}(\infty)=0, \theta(\infty)=0, g(\infty) \rightarrow 0 .
\end{aligned}
$$

Where $\xi=\zeta\left(T_{w}-T_{\infty}\right)$ defines the viscosity parameter, $h=\epsilon\left(T_{w}-T_{\infty}\right)$ describes the thermal conductivity term, $K=\kappa / \mu_{B_{\infty}}$ indicates the micropolar material parameter, $D a=\frac{\vartheta}{a K_{p}}$ denotes the Darcy parameter, $M=\frac{\sigma B_{0}^{2}}{a \rho_{\infty}}$ defines the magnetic field term, $\operatorname{Pr}=\frac{\mu_{\infty} C_{p}}{k_{\infty}}$ is the Prandt number, $E c=\frac{u_{w}^{2}}{C_{p}\left(T w-T_{\infty}\right)}$ indicates the Eckert number and $B=\frac{Q^{\star}}{a C_{p}}$ describes the heat source parameter. The following quantities namely; the skin friction coefficient and the local Nusselt number draw the attention of the engineering community. These quantities are respectively described as:

$$
C_{f x}=\frac{\tau_{w}}{\rho_{\infty} U_{w}^{2}}, N u_{x}=\frac{x q_{w}}{k\left(T_{w}-T_{\infty}\right)}
$$

where $\tau_{w}$ indicates the shear stress while $q_{w}$ defines the heat flux at the surface. Here,

$$
\tau_{w}=\left(\mu+\frac{P_{y}}{\sqrt{2 \pi_{c}}}\right) \frac{\partial u}{\partial y}+\left.\kappa N\right|_{y=0}, q_{w}=-\left.\left(k_{\infty}+\frac{16 T^{3} \sigma^{\star}}{3 k^{\star}}\right) \frac{\partial T}{\partial y}\right|_{y=0},
$$

in view of equations (13) and (19), the quantities in (18) orderly yields (20-21)

$$
\begin{gathered}
C_{f x}=\left(\left(1+\frac{1}{\beta}\right)(1+(1-g) K)\right) R e_{x}^{-1 / 2} f^{\prime \prime}(0) \\
N u_{x}=-[1+R(1+\theta(0))] R e_{x}^{1 / 2} \theta^{\prime}(0)
\end{gathered}
$$




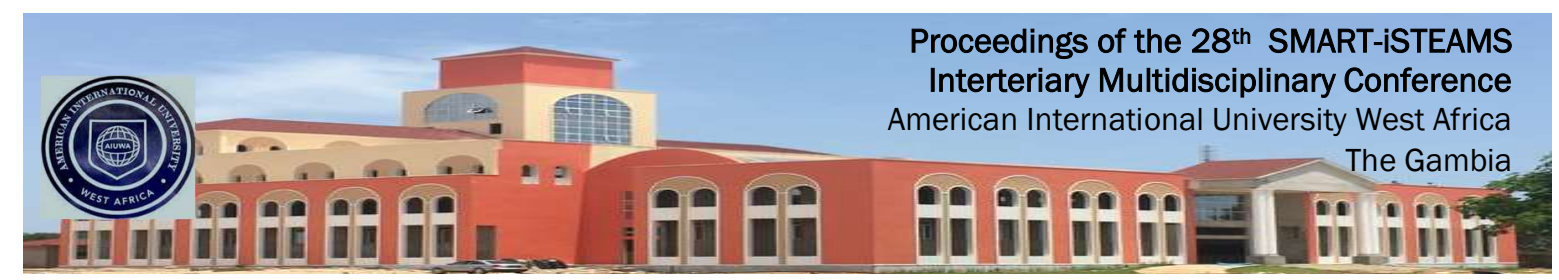

\section{NUMERICAL METHOD WITH VALIDATION}

The system of Eqs. (14-17) constitutes a nonlinear boundary value problem which the solution has been sought numerically using the shooting technique alongside with Runge-Kutta-Fehlberg scheme. Due to its popularity in the open literature, we do communicate the detail description in this study. The details can be found in the work of (see Ali, 1994, Attili and Syam, 2008; Xu and Lee, 2013; Mabood and Das, 2016; Mahanthesh et al., 2018;).

The default values used in this study are listed as $K=M=D a=0.5, R=h=\xi=B=$ $0.2, E c=0.1, n=0.5$ and $\operatorname{Pr}=0.72$ unless otherwise specified in the graphs. To authenticate the accuracy of our numerical solution a comparison is carried out in Table 1 with some existing results in literature in the limiting condition and both solutions are found in good harmony. The comparison is carried out with the work of Chen (1998) and Qasim et al. (2013) for different values of Prandtl number $\operatorname{Pr}$ when $K=E c=R=D a=M=h=\xi=B=0$ and $\beta \rightarrow \infty$. The highest percentage relative difference is found to be $0.42 \%$.

Table .1: Comparison of the values of the Nusselt number $N u_{x}$ with existing studies for various values of $\mathrm{Pr}$

\begin{tabular}{|c|c|c|c|c|c|c|}
\hline$P r$ & Chen (1998) & $\begin{array}{l}\text { Present } \\
\text { study }\end{array}$ & $|\% R D|$ & $\begin{array}{c}\text { Qasim e al. } \\
\text { (2013) }\end{array}$ & $\begin{array}{l}\text { Present } \\
\text { study }\end{array}$ & $\mid \% R D$ \\
\hline 0.72 & 0.46170 & 0.46368 & 0.42 & 0.46360 & 0.46368 & 0.02 \\
\hline 1.00 & 0.58010 & 0.58211 & 0.35 & 0.58202 & 0.58211 & 0.01 \\
\hline 3.00 & 1.16525 & 1.16535 & 0.01 & 1.16525 & 1.16535 & 0.01 \\
\hline 5.00 & 1.56805 & 1.56816 & 0.01 & 1.56805 & 1.56816 & 0.01 \\
\hline 7.00 & 1.89540 & 1.89551 & 0.01 & 1.89542 & 1.89551 & 0.00 \\
\hline 10.00 & 2.30800 & 2.30811 & 0.00 & 2.30800 & 2.30811 & 0.00 \\
\hline 100.00 & 7.76565 & 7.76576 & 0.00 & 7.75826 & 7.76576 & 0.10 \\
\hline
\end{tabular}




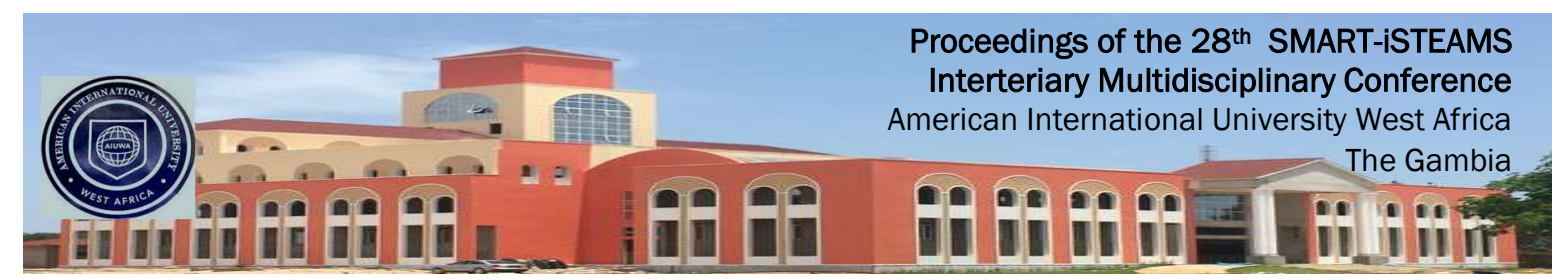

Table 2: Computational values of the values of $C_{\mathrm{fx}}$ with variation in $K, \beta, \xi, D a$ and $M$

\begin{tabular}{|c|c|c|c|c|c|}
\hline$K$ & $\beta$ & $\xi$ & $D a$ & $M$ & $C_{f x}$ \\
\hline $\begin{array}{l}0.1 \\
0.3 \\
0.5\end{array}$ & 0.1 & 0.2 & 0.4 & 0.5 & $\begin{array}{l}0.6244414 \\
0.5454724 \\
0.4907923\end{array}$ \\
\hline 0.3 & $\begin{array}{l}0.1 \\
0.3 \\
0.5\end{array}$ & 0.2 & 0.4 & 0.5 & $\begin{array}{l}0.6675712 \\
0.7892711 \\
0.8686437\end{array}$ \\
\hline & & $\begin{array}{l}0.1 \\
0.3 \\
0.5\end{array}$ & & & $\begin{array}{l}0.6574750 \\
0.6772104 \\
0.6952372\end{array}$ \\
\hline & & 0.2 & $\begin{array}{l}0.1 \\
0.3 \\
0.7\end{array}$ & & $\begin{array}{l}0.4484146 \\
0.6046815 \\
0.8249991\end{array}$ \\
\hline & & & 0.4 & $\begin{array}{l}0.3 \\
0.6 \\
0.8\end{array}$ & $\begin{array}{l}0.6571026 \\
0.6727449 \\
0.6829753\end{array}$ \\
\hline 0.0 & $\infty$ & $\begin{array}{l}0.1 \\
0.3 \\
0.5\end{array}$ & & 0.5 & $\begin{array}{l}1.3827854 \\
1.3926768 \\
1.4026076\end{array}$ \\
\hline & & 0.2 & $\begin{array}{l}0.1 \\
0.3 \\
0.7\end{array}$ & & $\begin{array}{l}1.2739253 \\
1.2572062 \\
1.4920829\end{array}$ \\
\hline & & & 0.4 & $\begin{array}{l}0.3 \\
0.6 \\
0.8\end{array}$ & $\begin{array}{l}1.3144136 \\
1.3219056 \\
1.4909093\end{array}$ \\
\hline
\end{tabular}

\section{RESULTS AND DISCUSSION}

This section presents the graphical illustrations and the discussion of the effects of various physical parameters on the non-dimensional quantities namely: velocity, microrotation and temperature profiles.. Besides, the reaction of the skin friction coefficient $C_{f x}$ to variations in some selected parameters, namely, micropolar parameter, Casson fluid parameter $\beta$, viscosity term $\xi$, Darcy number $D a$ and magnetic field parameter $M$ are recorded in Table 2 . From this table, it is clearly noticed that an increase in $K$ facilitates the reduction in the viscous drag such that $C_{f x}$ is drastically reduced. However, there is a spontaneous increase in the skin friction coefficient $C_{f x}$ as the value of $\beta, \xi D a$ and $M$ improves. In the absence of the non-Newtonian fluids $(K=0, \beta \rightarrow \infty)$, the fluid becomes Newtonian. In this case, the skin friction coefficient is 


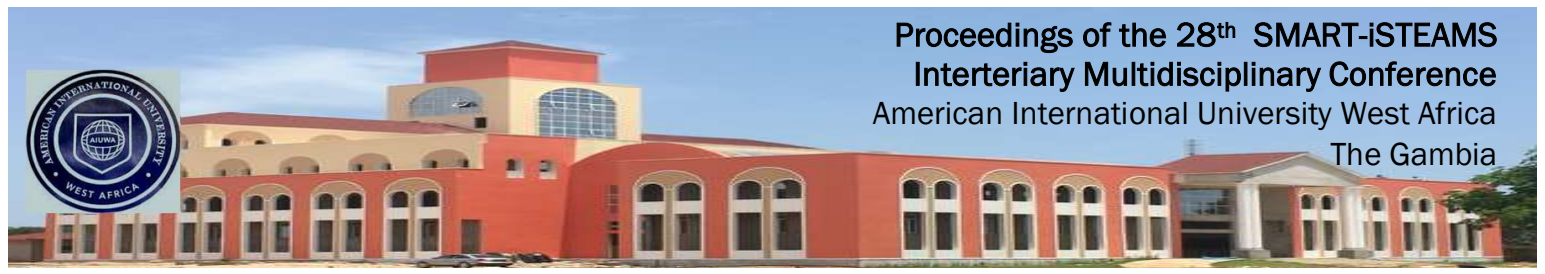

examined and it is observed that the skin friction coefficient is higher for growth in $\xi, D a$ and $M$ as compared to the case of non-Newtonian fluid as noticed from the table..

Figure 2 portrays the reaction of the velocity profile for variations in the viscosity parameter $\xi$ in the presence of the Casson fluid parameter $\beta$.. Increasing the magnitude of $\xi$ makes the fluid to be more viscous and thereby creates higher resistance to the fluid motion and thereby decelerates the flow as seen in this figure. Similarly, there is a reduction in the fluid flow owing to a rise in $\beta$. This trend indicates that growth in $\beta$ dictates a fall in the velocity field due to a reduction in the yield stress as $\beta$ rises which in turn decelerates the motion of the fluid. In addition, a rise in $\beta$ empowers the plastic dynamic viscosity over the Casson fluid viscosity and at such, the flow is resisted. However, the temperature field reacts conversely with a rise in the viscosity parameter $\xi$ and $\beta$ as displayed in Fig. 3. The thermal boundary layer thickens with an increase in $\xi$ and in consequence, the temperature is raised. The resistance created due to fluid viscosity develops a frictional heating in the flow regime and thus provides additional heating leading to a rise in temperature. The microrotation of the fluid parcels also enhances due to a rise in $\xi$ as found in Fig. 4.

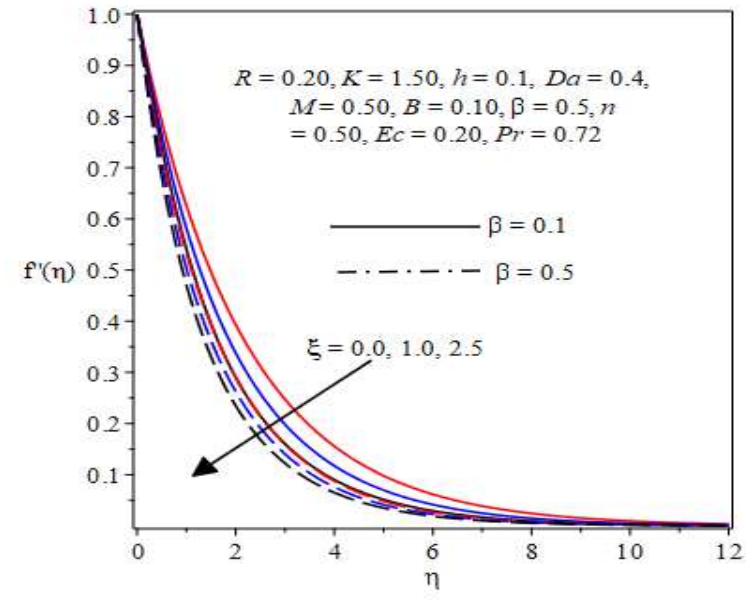

Fig. 2 Effect $\xi$ and $\boldsymbol{\beta}$ on velocity profile

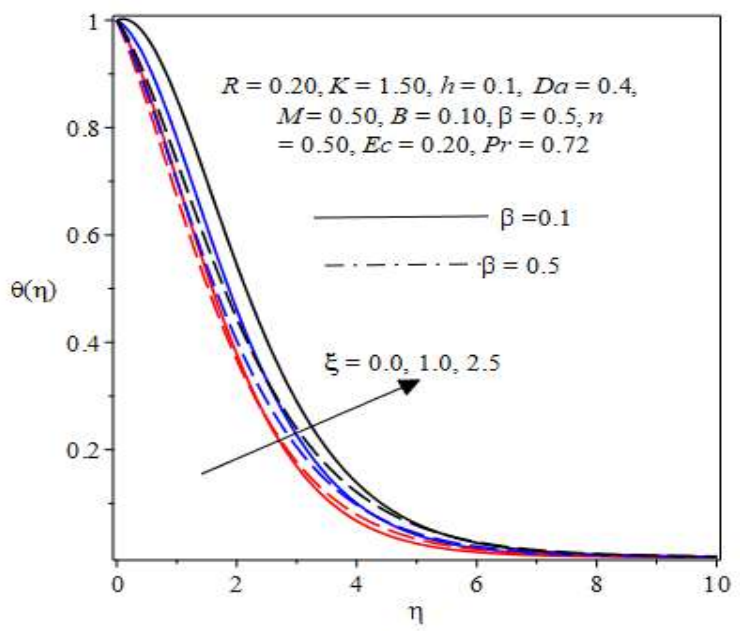

Fig. 3 Effect of $\xi$ and $\boldsymbol{\beta}$ on temperature

Figure 5 depicts the influence of material micropolar parameter $K$ on the velocity profile in the existence of the magnetic field term $M$. Clearly, the hydrodynamic boundary layer grows with rising $K$. A rise in $K$ depicts a fall in the in the dynamic viscosity and a rise in the microrotation viscosity $\kappa$ and thus, the viscous force is reduced and the flow increases. On the hand, a rise in $M$ decelerates the motion of the fluid due to the drag created by the Lorentz force by the imposition transverse magnetic field to an electrically conducting micropolar-Casson. The Lorentz force offers a drag in the fluid motion with the corresponding increase in $M$ and hence, the fluid velocity reduces. The temperature profiles fall for a rise in $K$ but develops with growth in $M$ due to friction in the fluid particles as a result of resistance created by the Lorentz force as seen in Fig. 6 . The microrotation field shrinks with an increase in $K$ but grows with a ruse in the magnitude of $M$ as shown in Fig. 7 . The microrotation field is augmented due to magnetic field influence. 


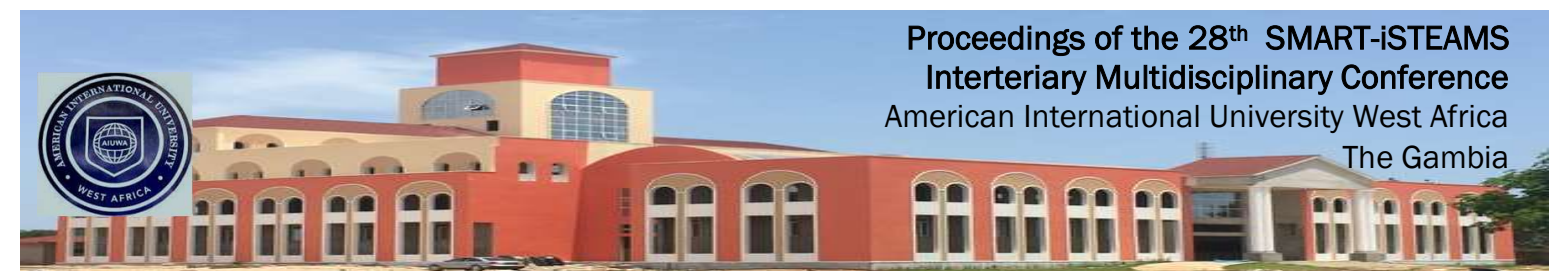

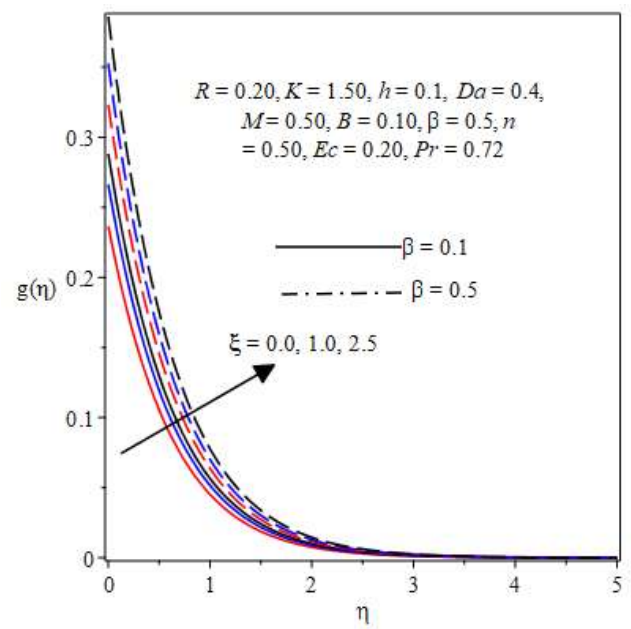

Fig. 4 Effects $\xi$ and $\beta$ on microrotation

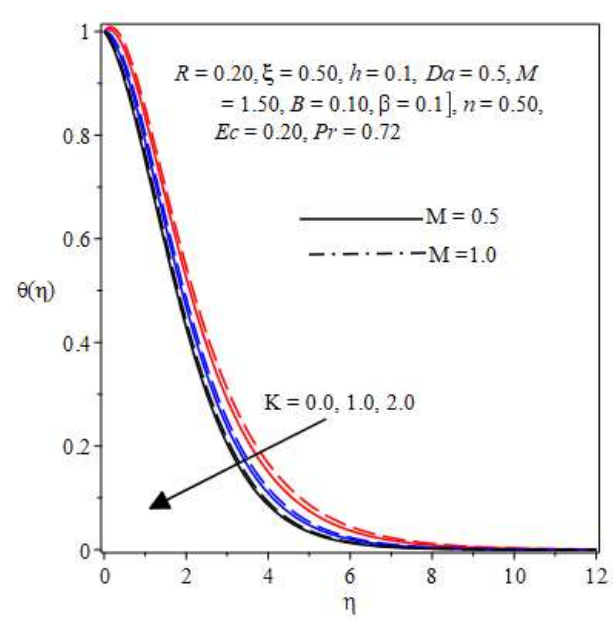

Fig. 6 Graph of $K$ and $M$ on temperature

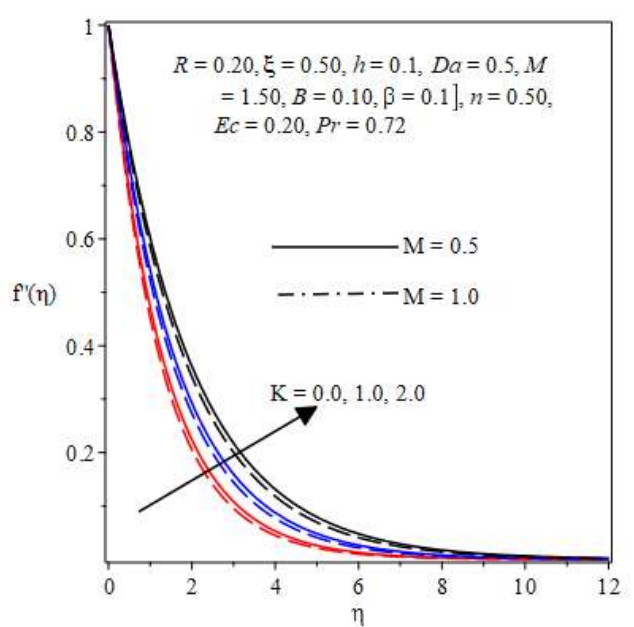

Fig. 5 Graph of $K$ and $M$ on velocity

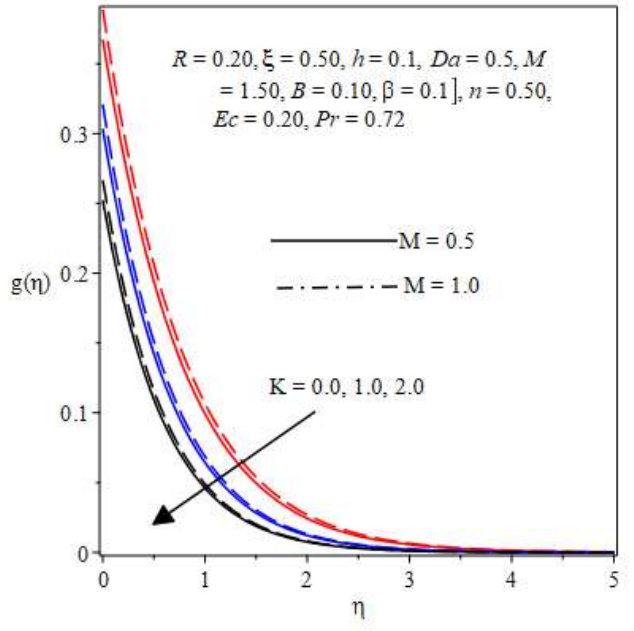

Fig. 7 Plot of $K$ and $M$ on microrotation 


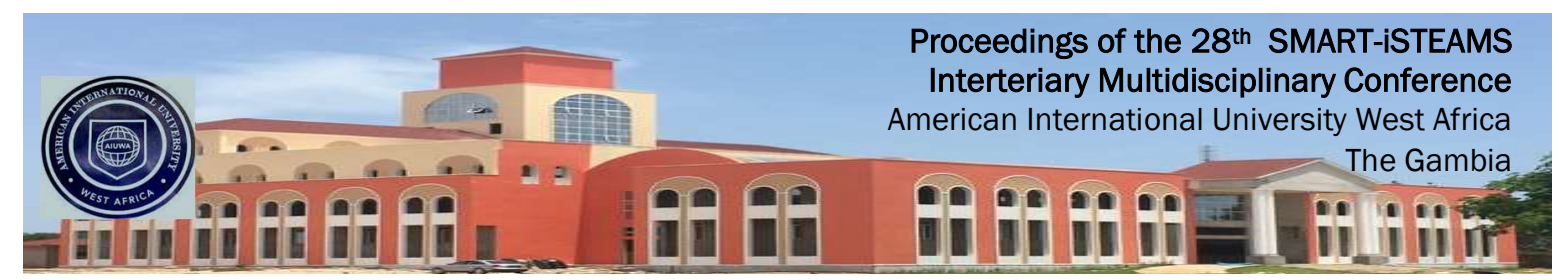

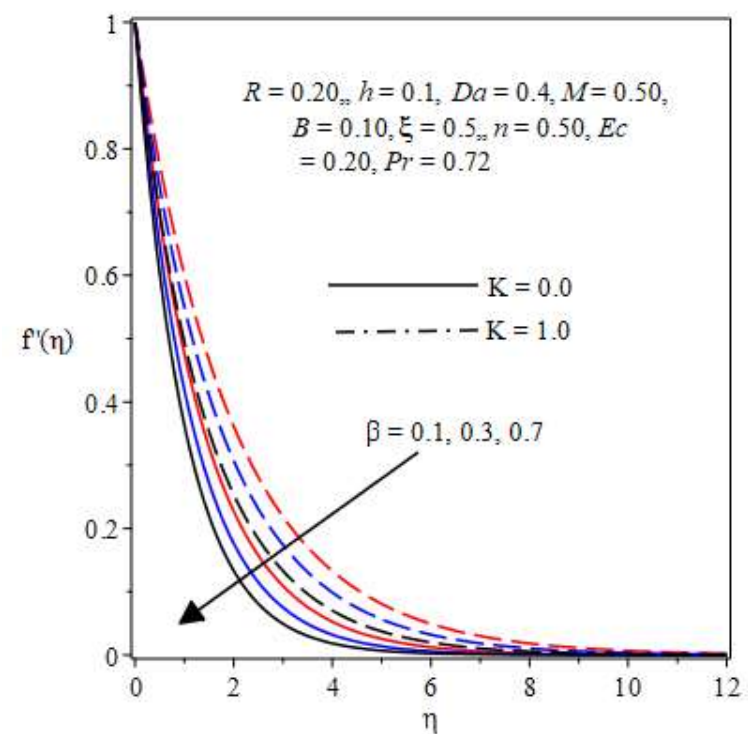

Fig. 8 Impact $\boldsymbol{\beta}$ and $\boldsymbol{K}$ on velocity

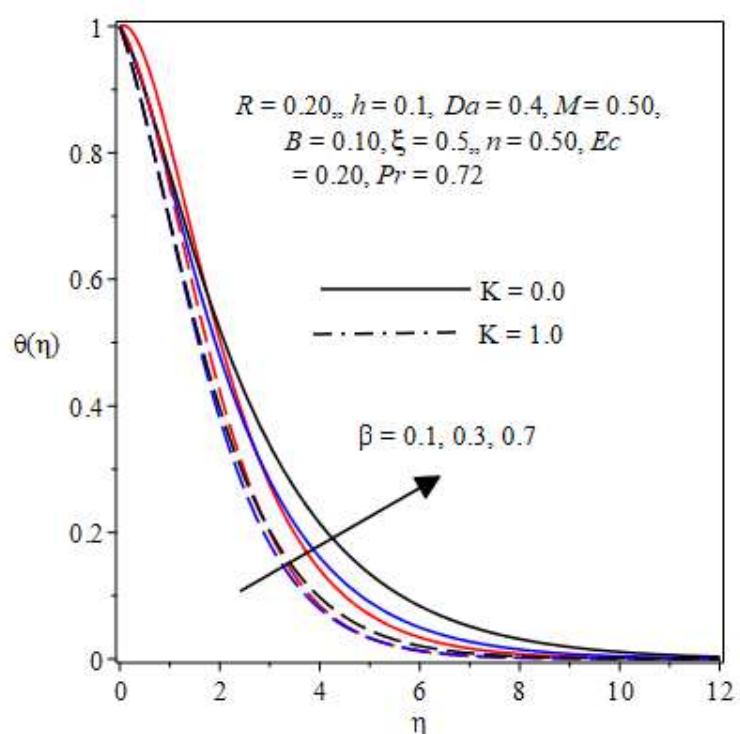

Fig. 9 Plot of $\boldsymbol{\beta}$ and $\boldsymbol{K}$ on temperature

The combined effects of micropolar-casson fluid on the velocity and temperature profiles are described in Figs. 8 and 9 respectively. Fig. 8 reveals the shrinking nature of the momentum boundary layer structure with a raise in the magnitude of $\beta$ in the presence or absence of the micropolar fluid influence. However, the micropolar fluid influence boosts the velocity profile. The heat distribution appreciates as $\beta$ grows as noted in Fig. 9. Meanwhile, the heat energy is lower in the presence of micropolar fluid material term $K$ as compares to its presence.

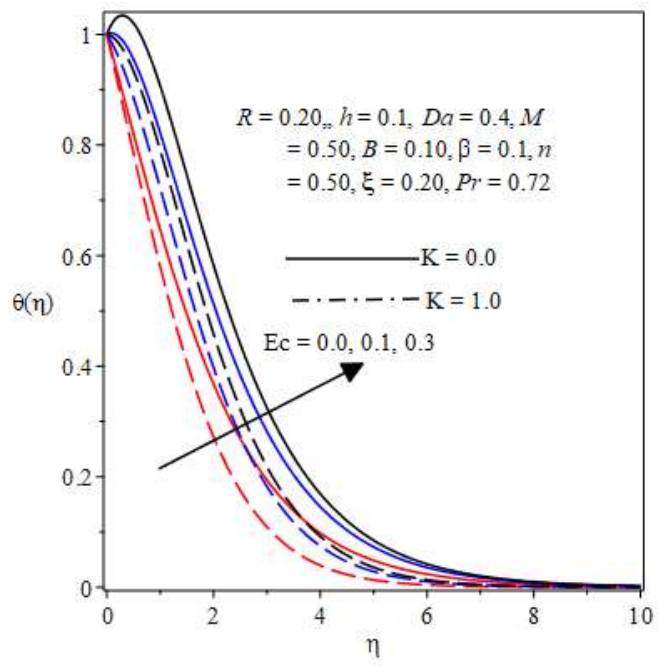

Fig. 10 Effects $\boldsymbol{E} \boldsymbol{c}$ and $\boldsymbol{K}$ on temperature

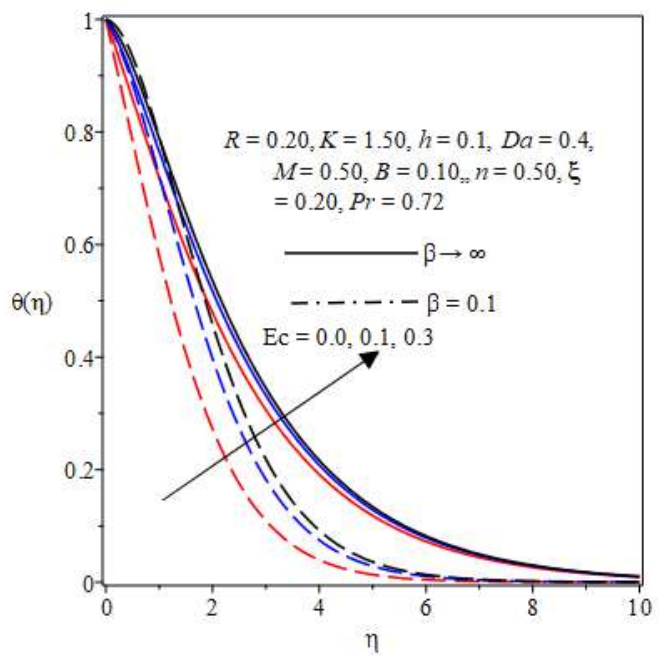

Fig. 11 Effect of $\boldsymbol{E} \boldsymbol{c}$ and $\boldsymbol{\beta}$ on temperature 


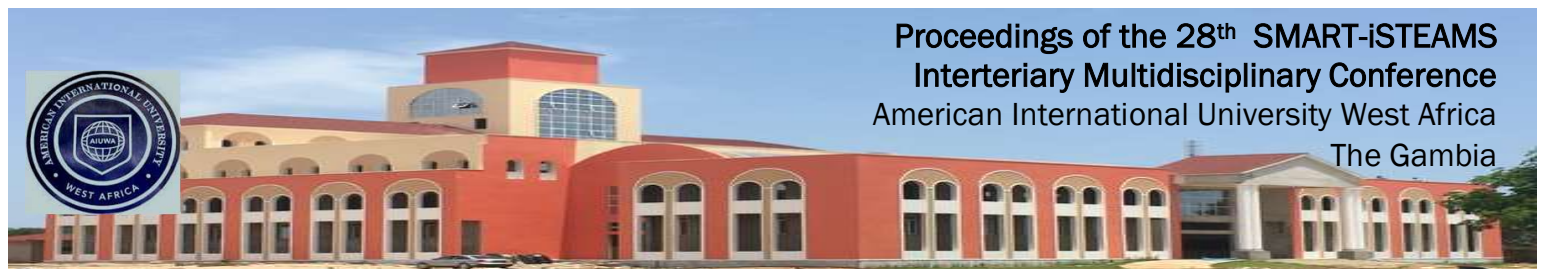

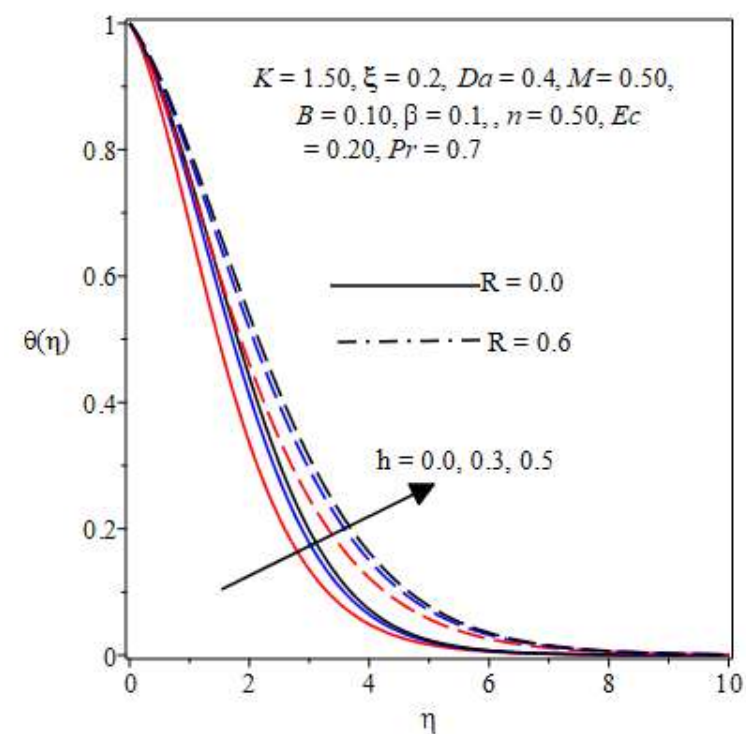

Fig. 12 Effects $\boldsymbol{h}$ and $\boldsymbol{R}$ on temperature

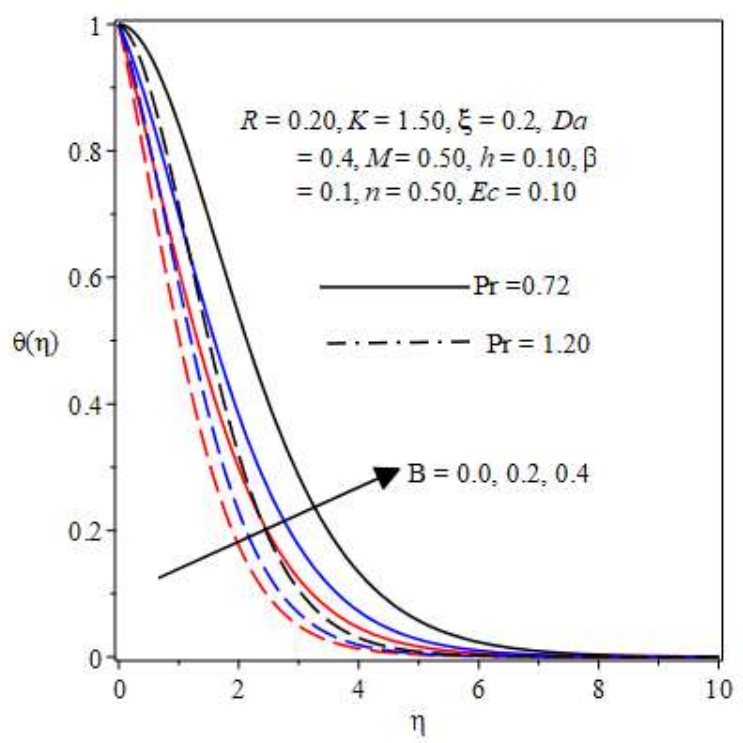

Fig. 13 Effects $\boldsymbol{B}$ and $\boldsymbol{P r}$ on temperature

Figures 10-11 respectively reveals the reaction of temperature profile to variation in the Eckert number $E c$ in the presence of material micropolar term $K$ and in the presence of Casson fluid term $\beta$. Clearly, the thermal field soars in both cases as Ec rises in magnitude. This is due to the frictional heating on the account of friction between the fluid particles and the stretching sheet. However, the impact of $K$ and $\beta$ are opposite each other as shown in these figures. Similarly, the thermal field appreciates due to growth in the thermal conductivity $h$ and radiation $R$ terms as illustrated in Fig. 12. The heat source term $B$ also boost the temperature profile due to additional heating as displayed in Fig. 13. However, a rise in the Prandtl number Pr shrinks the thermal boundary layer and lowers the average temperature as portrayed in Fig. 13.

\section{CONCLUSION}

A numerical investigation has been carried out on the flow and heat transfer characteristics of magnetohydrodynamic micropolar-Casson fluid over a two-dimensional stretching sheet in a porous medium. The main equations of flow and heat dissipation are characterized by variable viscosity and thermal conductivity, thermal radiation, viscous dissipation and internal heat source. Similarity transformations variables are used to re-modelled the governing equations which are then solved by shooting technique associated with Runge-Kutta Fehlberg integration scheme. The obtained results strongly agree with published works in the related studies in literature as special cases of the present study. The impact of the physical parameters are shown in the various graphs and deliberated. Conclusively, it is revealed from the study that: 


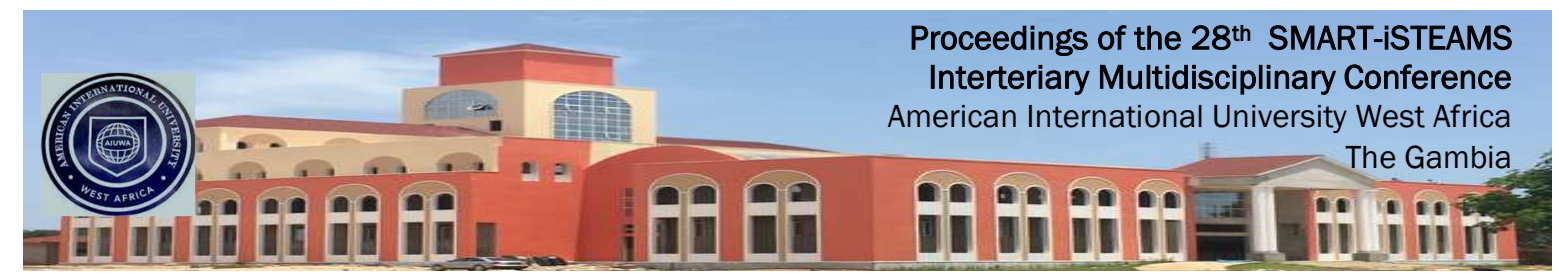

* There is a decelerated flow in the presence of Casson fluid $\beta$, viscosity parameter $\xi$. Growth in the Casson fluid parameter compels a rise in the viscous force due to a decrease in the yield stress as $\beta$ grow leading to a resistance in the fluid motion.

* The micropolar material parameter $K$ enhances the fluid motion by the reduction in the strength of the dynamic plastic viscosity and a rise in the vortex viscosity. Conversely, the thermal field falls with growing values of $K$ leading to a cool surface.

* The thermal boundary layer expands with growth in the viscosity parameter $\xi$ and Casson fluid material parameter $\beta$ and thus, the surface temperature improves as $\xi$ uplifts, a frictional heating is developed in the flow regime which offers extra heating in the thermal field.

* The surface temperature rises by a rise in Ec, radiation term $R$ and internal heat source $B$ but an increase in the Prandtl number $P r$ behaves contrary on the thermal field. The temperature can be cooled in the presence of $\mathrm{Pr}$.

\section{REFERENCES}

1. Ahmad, K., Hanouf, Z. and Ishak, A. (2016). Mixed convection Jeffrey fluid flow over an exponentially stretching sheet with magnetohydrodynamic effect, AIP ADVANCES 6, 18, 035024.

2. Alkasasbeh, H. T. (2018). Numerical Solution of Micropolar Casson Fluid Behaviour on Steady MHD Natural Convective Flow about A Solid Sphere. Journal of Advanced Research in Fluid Mechanics and Thermal Sciences 50(1), 55-66.

3. Ali, M. E. (1994). Heat transfer characteristics of a continuous stretching surface, Warme-und Stoffubertragung, 29(4), 227-234.

4. Animasaun I.L and Aluko O.B. (2014). Analysis on variable fluid viscosity of non-Darcian flow over a moving vertical plate in a porous medium with suction and viscous Dissipation. International Organization of Scientific Research (Journal of Engineering), 2014, 18-32.

5. Animasaun, I. L. (2014). Effects of thermophoresis, variable viscosity and thermal conductivity on free convective heat and mass transfer of non-Darcian MHD dissipative Casson fluid flow with suction and nth order of chemical reaction, Journal of Nigeria Mathematical Society, 2014, 1-21.

6. Attili, B. S. and Syam, M. L. Efficient shooting method for solving two point boundary value problems, Chaos, Solitons and Fractals, 35(5), 895-903, (2008).

7. Bird, R.B., Dai, G.C., Yarusso, B.J. (1983). The rheology and flow of viscoplastic materials. Rev Chem Eng, 1, 1-83.

8. Casson, N. (1959). Rheology of Disperse Systems in Flow Equation for Pigment Oil Suspensions of the Printing Ink Type, Rheology of disperse systems, C. C. Mill, Ed., 84102, Pergamon Press, London, U. K. (1959).

9. Chen, J., Liang, C. and Lee J. D. (2011). Theory and simulation of micropolar fluid dynamics. J. Nanoengineering and Nanosystems, 224, 31-39.

10. Das, S., Mondal, H., Kundu, P. K. and Sibanda, P. (2018). Spectral quasilinearization method for Casson fluid with homogeneous heterogeneous reaction in presence of nonlinear thermal radiation over an exponential stretching sheet, Multidiscipline Modeling in Materials and Structures, https://doi.org/10.1108/MMMS-04-2018-0073 


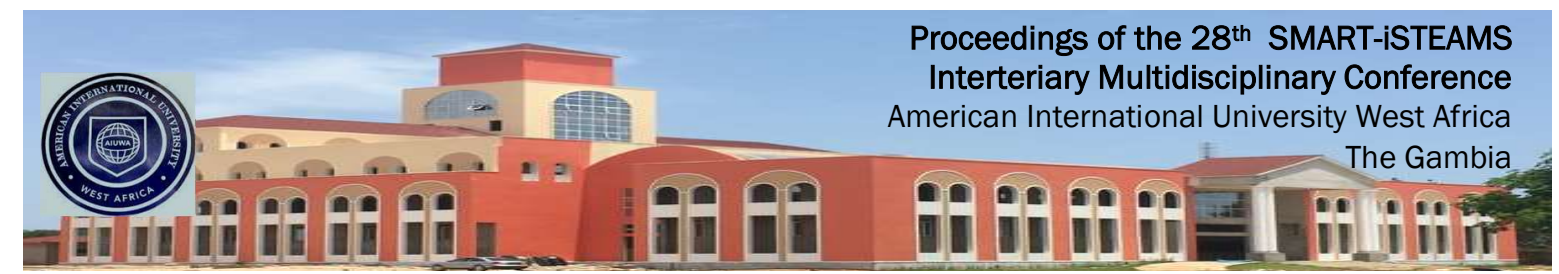

11. Eringen, A. C. (1964). Simple microfluids. \{International Journal of Engineering Science, 2(2), 205-217.

12. Eringen, A. C. (1966). Theory of micropolar fluids. J. Math. Anal. Appl., \{16\}, 1-18.

13. Fatunmbi, E. O. and Adeniyan, A. (2018). MHD stagnation point-flow of micropolar fluid past a permeable stretching plate in porous media with thermal radiation, chemical reaction and viscous dissipation. Journal of Advances in Mathematics and Comp Science, $\{26\}(1), 1-19$.

14. Fatunmbi, E. O. and Fenuga, O. J. (2017). MHD micropolar fluid flow over a permeable stretching sheet in the presence of variable viscosity and thermal conductivity with Soret and Dufour effects. International Journal of Mathematical Analysis and Optimization: Theory and Applications, \{2017\}\}, 211- 232.

15. Fatunmbi, E. O. and Adeniyan, A. (2020). Nonlinear thermal radiation and entropy generation on steady flow of magneto-micropolar fluid passing a stretchable sheet with variable properties, Results in Engineering, 6, 100142, 1-10.

16. Fatunmbi, E. O. and Okoya, S. S. (2020). Heat transfer in boundary layer magnetomicropolar fluids with temperature-dependent material properties over a stretching sheet, Advances in Materials Science and Engineering, 2020, 1-11.

17. Fatunmbi, E. O. and Okoya, S. S. (2020). Heat transfer in boundary layer magneto-micropolar fluids with temperature-dependent material properties over a stretching sheet, Advances in Materials Science and Engineering, 2020, 1-11. Diffusion Foundations. 26, 63-77.

18. Fatunmbi, E. O. and Okoya, S. S. (2021). Quadratic Mixed Convection Stagnation-Point Flow in Hydromagnetic Casson Nanofluid over a Nonlinear Stretching Sheet with Variable Thermal Conductivity, Defect and Diffusion, 409, 95-109.

19. Gbadeyan, J. A., Titiloye, E. O., Adeosun, A. T. (2020). Effect of variable thermal conductivity and viscosity on Casson nanofluid flow with convective heating and velocity slip, Heliyon, 6, 1-10, e03076.

20. Ibrahim, W. and Makinde, O. D. (2015). Magnetohydrodynamic Stagnation Point Flow and Heat transfer of Casson nanofluid past a stretching sheet with slip and convective boundary condition, Journal of Aerospace Engineering, 1-11.

21. Iqbal, Z., Mehmood, R. Azhara, R. and Mehmood, Z. (2017). Impact of inclined magnetic field on micropolar Casson fluid using Keller box algorithm. Eur. Phys. J. Plus, 132, 113.

22. Krishna, V. H., Reddy, G. V. R. and Makinde, O. D. Chemical Reaction Effect on MHD Flow of Casson Fluid with Porous Stretching Sheet, Defect and Diffusion, 389, 100-109.

23. Layek, G.C, Mukhopadhyay, S, Samad, S.K. (2007). Heat and mass transfer analysis for boundary layer stagnation point flow towards a heated porous stretching sheet with heat absorption/generation and suction/blowing. Int Commun Heat Mass Transfer, ;34, 347-56

24. Lukaszewicz, G. (1999). \{\it Micropolar fluids: Theory and Applications\} (1st Ed.). Birkhauser, Boston.

25. Mabood, F. and Das, K. (2016). Melting heat transfer on hydromagnetic flow of a nanofluid over a stretching sheet with radiation and second order slip, Eur. Phys. J. Plus 131(3). 


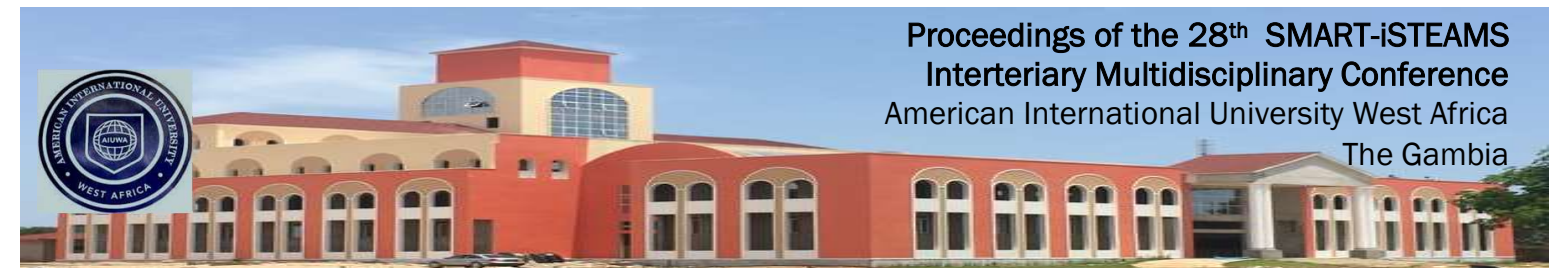

26. Mahanthesh, B., Gireesha, B. J., Gorla, R. S. R. and Makinde, O. D. (2018). Magnetohydrodynamic three-dimensional flow of nanofluids with slip and thermal radiation over a nonlinear stretching sheet: a numerical study, Neural Comput \& Applic, 30, 1557-1567.

27. Megahed, A. M. (2015). Effect of slip velocity on Casson thin film flow and heat transfer due to unsteady stretching sheet in presence

28. Mahmoud, M. A. A. (2007). Thermal radiation effects on MHD flow of a micropolar fluid over a stretching surface with variable thermal conductivity Physica A , \{375, 401-410.

29. Mehmood, Z. Mehmood, R., and Z. Iqbal (2017). Numerical investigation of micropolar Casson fluid over a stretching sheet with internal heating, Communications in Theoretical Physics, 67(4), 443-448.

30. Mitsoulis, E.(2007). Flows of viscoplastic materials: Models and computations. The British society of rheology, 2007, 135-78

31. Omotola, O. E. and Fatunmbi, E. O. (2021). Magnetohydrodynamic radiative Casson fluid motion past a convectively heated and slippery non-linear permeable stretching plate, Fepi-Jopas, 3(1), 45-55.

32. Qasim, M., Khan, I. and Shafle, S. (2013). Heat transfer in a micropolar fluid over a stretching sheet with Newtonian heating. Plos One, 8(4), 1-6.

33. Salem, A. M, Fathy, R. (2012). Effects of variable properties on MHD heat and mass transfer flow near a stagnation point towards a stretching sheet in

34. SallehMZ, Nazar R, Pop I. (2010) Boundary layer flow and heat transfer over a stretching sheet with Newtonian heating. J Taiwan Inst Chem Eng, 41, 651-655.

35. Ullah, Z, Zamana, G. and Ishak, A. (2020). Magnetohydrodynamic tangent hyperbolic fluid flow past a stretching sheet, Chinese Journal of Physics, 66 258-268.

36. Vajravelu, K., Prasad, K. V., Vaidya, H., Basha, N. Z. and Chiu-On Ng. (2016). Mixed Convective Flow of a Casson Fluid over a Vertical Stretching Sheet, Int. J. Appl. Comput. Math, 1-20. DOI 10.1007/s40819-016-0203-6

37. Xu, L. and Lee, E.W.M. Variational iteration method for the magnetohydrodynamic flow over a nonlinear stretching sheet. Abst Appl Anal 5 pages (2013). 